Carmona Santiago, J., García-Ruiz, M., Maíquez, L. y Rodrigo López, M.J. (2021). Las expectativas y metas de las familias gitanas en Canarias hacia el éxito escolar . Revista de Investigación Educativa, 39(1), 71-89. DOI: http://dx.doi.org/10.6018/rie.400901

\title{
Las expectativas y metas de las familias gitanas en Canarias hacia el éxito escolar
}

\section{The expectations and goals of Romani families in the Canary Islands regarding school success}

\author{
José Carmona Santiago*, Marta García-Ruíz**, Mª Luisa Máiquez*, María José Rodrigo López* \\ * Facultad de Psicología y Logopedia, Universidad de La Laguna \\ ** Facultad de Ciencias Políticas, Sociales y de la Comunicación, Universidad de La Laguna
}

\begin{abstract}
Resumen
La población romani/gitana en España oscila entre las 725.000 y las 750.000 personas. Sin embargo, solo el 22,2\% del alumnado gitano logra acabar con éxito la Educación Secundaria Obligatoria, mientras que un $75,6 \%$ de la sociedad mayoritaria lo logra. A partir de una muestra de 90 familias gitanas, con hijos e hijas estudiantes entre 6 y 17 años residentes en las Islas Canarias, se analizan los deseos, expectativas, celebraciones más importantes y metas de los padres y madres gitanas respecto al futuro de sus hijos e hijas. La recogida de información se ha realizado a través de dos entrevistas semiestructuradas siendo el procedimiento de análisis la Teoría Fundamentada. Tras el proceso de selección se han identificado 15 familias, con metas orientadas al avance educativo de sus hijos e hijas, a las que se examinan las fortalezas, oportunidades, debilidades y amenazas que perciben. Este grupo de familias ha experimentado una buena relación con la escuela, se interesan y apoyan las tareas escolares, dando un paso hacia delante en la promoción de la formación académica de sus hijos e hijas. Son familias referentes, capaces de tener coherencia entre sus deseos, expectativas, celebraciones y prioridades; a lo que se le suma su destreza para hacer frente a los desafíos diarios y muestran la capacidad de creer que un futuro mejor es posible para sus hijos e hijas mientras luchan para conseguirlo.

Palabras claves: gitano integración escolar; necesidad de educación; planificación familiar.
\end{abstract}

Correspondencia: José Carmona Santiago, alu0100708653@ull.edu.es, Facultad de Psicología y Logopedia, Universidad de La Laguna, Campus de Guajara, s/n, 38071 San Cristóbal de La Laguna, Santa Cruz de Tenerife 


\begin{abstract}
The Romani population in Spain ranges between 725,000 and 750,000 people. However, only 22.2\% of gypsy students successfully finish Compulsory Secondary Education, while $75.6 \%$ of majority-population students manage to complete their studies. Drawing from a selected sample of 90 Romani families with sons and daughters aged 6-17 living in the Canary Islands, we analyse the most important wishes, expectations, celebrations and goals that Romani fathers and mothers share regarding the future of their children. Results have been collected using two semi-structured interviews, with Grounded Theory as our main research methodology. After the selection process, only 15 families with goals aimed at the educational advancement of their children were identified, families who perceive and experience specific strengths, opportunities, weaknesses and threats examined in this article. This group of families enjoy a positive relationship with the school, showing interest, support and caring about their children's school tasks. It is this commitment that has helped them take a step forward in the promotion of their children's academic training. They are referents, their wishes, expectations, celebrations and priorities being consistent with one another. They also have the ability to face daily challenges and believe that a better future for their children is possible, even as they struggle to make it happen.
\end{abstract}

Keywords: gipsy; school integration; educational needs; family planning.

\title{
Introducción
}

El Pueblo Gitano o romaní es una minoría étnica trasnacional con una población que se estima entre los 10 a 12 millones de personas en Europa, siendo el grupo social más rechazado y estigmatizado del continente (Fernández-Jiménez, 2018; Parra et al., 2017). En España, el Pueblo Gitano se concibe actualmente como un colectivo culturalmente diverso que convive como minoría cultural en todas las Comunidades Autónomas del Estado español (Macías, 2017). Según los datos aportados por Laparra (2011), la población gitana oscila entre las 725.000 y las 750.000 personas. Mientras que en el resto de los países europeos la infancia romaní tiene acceso limitado a la educación y sufre una exclusión sistemática de las escuelas (Jiménez-González, 2018; Sime et al., 2014), en España las familias gitanas han avanzado en la escolarización de sus hijos e hijas. Hay que destacar que el 96\% de los menores gitanos está matriculado en la escolarización obligatoria (Ministerio de Sanidad, Servicios Sociales e Igualdad, 2016) y el 87\% de la infancia ha asistido a Educación Infantil Si décadas atrás llevar a los hijos a la escuela suponía una amenaza de "apayamiento", ahora se ha transformado en un símbolo de prestigio (Salinas, 2009). Sin embargo, únicamente el 17\% de las personas gitanas tienen estudios de Secundaria Obligatoria o superiores, frente al 77\% del conjunto de la población general, lo que evidencia la brecha existente (Enseñanza CCOO, 2019; Fundación Secretariado Gitano, 2019).

El Comité de Ministros del Consejo de Europa (2000, citado en Fundación Secretariado Gitano, 2000) ha reconocido que la situación del alumnado gitano es el resultado de viejas políticas que han conducido a la asimilación o bien a la segregación, considerándolos "discapacitados sociales y culturales". Como indica Salinas (2015), se trata de "seis siglos de conciudadanía accidentada" (p.96). Siguiendo la teoría ecológico- 
sistémica de Bronfenbrenner sobre el desarrollo humano (1987), las desigualdades y la marginación de la etnia gitana se habrían producido en el macrosistema, ya que se trata de valores, ideologías y políticas públicas equivocadas sostenidas por la sociedad mayoritaria durante años que moldean la vida de los individuos que nacen y crecen en ese entorno. También habría que hacer alusión al exosistema, es decir, al conjunto de influencias negativas que se derivan de la inadecuación de los recursos de apoyo y la baja calidad de vida en el ámbito de los barrios (en gran parte marginales), así como de los escasos esfuerzos para la integración laboral y social de las familias de etnia gitana.

Si bien es necesario seguir avanzando en la creación de políticas y estrategias que actúen a nivel de macrosistema y exosistema, también es clave promover cambios en el microsistema de la familia y su relación con el sistema escolar, ya que es en el seno de la familia donde se deben garantizar los derechos y el desarrollo integral de sus integrantes (Bronfenbrenner, 1987; Espinal et al., 2006; Rodrigo et al., 2010). Los estudios realizados principalmente desde la Sociología y la Antropología (Garriga y Carrasco, 2011; Giménez-Adelantado, 2003; López-Ros, 2011 y San Román, 2005) señalan que el Pueblo Gitano posee una serie de valores y rasgos identitarios comunes entre los que destacan los siguientes: la conciencia de un origen común; la salud y la libertad como valores deseados; la familia como uno de los ejes fundamentales sobre el que giran la mayor parte de sus valores; el respeto a la comunidad y hacia otras personas no gitanas, especialmente a personas mayores, mujeres y niños; y la empatía, la solidaridad, la hospitalidad, la ayuda y apoyo a toda familia gitana que lo necesite. Dichas señas de identidad constituyen un verdadero capital cultural (Yosso, 2006) que ha sido transmitido de generación en generación y sigue vivo por medio de la repetición de costumbres y pautas sociales, que se han interiorizado a través del proceso de socialización que realiza la familia y la propia comunidad con cada nuevo miembro del pueblo Caló (Giménez-Cortés et al., 2019; Kottak, 2011). Sin duda, la familia es para el Pueblo Gitano el pilar esencial sobre el que se fundamenta su cultura, cosmovisión, valores, arte y hasta la razón de su existencia (Gómez-Alfaro, 2010; Hancock, 2011).

Desde la psicología del desarrollo (Bornstein, 2002) así como desde la Declaración de los Derechos del Niño en 1959 y del reconocimiento de sus necesidades básicas (López, 2008) se ha llegado a un consenso sobre la "hoja de ruta" que garantiza un adecuado desarrollo del niño o de la niña cualquiera que sea su cultura. En consonancia, desde el ámbito de las políticas europeas sobre infancia y familia se ha señalado la importancia de apoyar a las figuras parentales para que puedan llevar a cabo un ejercicio adecuado de sus responsabilidades de modo que faciliten dicha hoja de ruta como garantía de su desarrollo. Así, desde la iniciativa de la parentalidad positiva (Consejo de Europa, Recomendación, 2006) se plantea el apoyo a las capacidades parentales destinadas a promover vínculos afectivos sanos, protectores y estables; proporcionar un entorno educativo estructurado en rutinas y hábitos que facilite la socialización; proporcionar estimulación, apoyo y oportunidades de aprendizaje; reconocer los logros y capacidades de los hijos e hijas y acompañarles en su vida cotidiana, todo ello en un entorno libre de violencia física, verbal y emocional (Rodrigo et al., 2015). En dicho modelo se da la bienvenida a la diversidad familiar y a las distintas formas de llevar a cabo el cumplimiento de dicha hoja de ruta con un gran respeto a las variaciones culturales siempre que no conculquen los derechos 
de la infancia. Ello supone que también se puede trasladar a las figuras parentales de las familias gitanas la responsabilidad de llevar a cabo dichas tareas así como la corresponsabilidad del estado para apoyar dicho ejercicio.

Por otro lado, como expone Salinas (2009), “del actual momento de escolarización generalizada de las niñas y niños gitanos parece que lo único que preocupa es el absentismo y el comportamiento disciplinario de algunas y algunos de ellos. Menos, su éxito escolar" (p. 167). Parece que el acento se pone en el déficit, potenciando el estereotipo negativo que arrastran las familias gitanas desde hace siglos. El éxito educativo de la infancia gitana demanda centrarse en soluciones y visibilizar qué hacen las familias que lo han logrado (Giménez-Adelantado, 2003). Si un 22,2\% del alumnado gitano ha cursado con éxito la Educación Secundaria Obligatoria (Ministerio de Sanidad, Servicios Sociales e Igualdad, 2012), el Pueblo Gitano cuenta con referencias positivas que demuestran que es posible. La investigación realizada por Abajo y Carrasco (2004), ha detectado factores claves para la continuidad académica del alumnado gitano, entre los que destacan la valoración positiva de sus posibilidades académicas por parte de los agentes de socialización, el apoyo y el compromiso sostenido del profesorado, el buen clima del centro educativo, la valoración y el apoyo de la familia, aspiraciones educativas, deseos por el progreso y el acceso a recursos educativos (referentes y apoyo) y económicos. Así mismo, el proyecto longitudinal INCLUD-ED, destaca que a medida que las familias gitanas son incluidas en el sistema educativo, contando con espacios dialógicos que facilitan el aprendizaje comunitario, mejora notablemente el rendimiento académico de la infancia gitana (Flecha y Soler, 2013). Desde una perspectiva más amplia, los estudios realizados en contextos de bajo nivel socioeconómico exponen como factores claves el nivel educativo de los padres, especialmente materno (Collet y Tort, 2011; Labin et al., 2019), las expectativas parentales (Álvarez et al., 2018), el entorno educativo familiar en forma de recursos disponibles (Marchesi y Martín, 2003), la cohesión familiar (Matamoros et al., 2017), el estilo educativo familiar (Kiernan y Mensah, 2011), vínculos de la familia con el entorno escolar (Collet y Tort, 2011) y la capacidad de resolución de conflictos familiares (Martínez et al., 2010).

En base a lo expuesto, el interés de este estudio radica en conocer cuál es la visión de futuro de sus hijos a hijas que tienen las familias del Pueblo Gitano que residen en la Comunidad Autónoma de Canarias. Concretamente interesa ahondar en el punto de vista de aquellas familias que están orientadas hacia el éxito escolar de sus hijos/ as, ya que se considera de vital importancia que se visibilice "la excepción" y no la "regla", a fin de identificar los elementos con los que cuentan aquellas familias que demuestran que el éxito escolar de la infancia gitana es posible. El caso de la población gitana residente en Canarias es especialmente paradigmático. Al no ser, en general, una población socialmente marginada se puede analizar de modo más puro aquellos condicionantes del éxito escolar que no provienen de esa situación de exclusión.

\section{Objetivos}

- Analizar las expectativas y metas de las figuras parentales respecto al futuro de sus hijos e hijas. 
- Identificar las características diferenciales de aquellas figuras parentales cuyos expectativas y metas están orientadas al éxito escolar en términos de las debilidades, amenazas, fortalezas y oportunidades percibidas en su consecución.

\section{Método}

El presente estudio es exploratorio-descriptivo. Se aplica una metodología cualitativa, que permite la comprensión de las personas dentro del marco de referencia de ellas mismas, entiende el contexto y a las personas bajo una perspectiva holística, sistémica e integradora, desde la cual la actitud del equipo investigador debe ser de apertura hacia el mundo del otro, reduciendo al máximo las subjetividades (Quecedo y Castaño, 2002). El conocimiento es el resultado de la interacción entre la realidad descrita por las familias gitanas y la exploración, comprensión, contrastación y sistematización de los datos aportados por ellas (Marcos et al., 2017). La orientación filosófica de esta metodología se enmarca en el constructivismo, entendiendo que la realidad no es estática, única y externa; sino más bien, subjetiva y global, dependiendo de quien la observa (Marcos et al., 2017).

El análisis de datos se ha realizado siguiendo el proceso metodológico de la Teoría Fundamentada (Grounded Theory; Glaser y Strauss, 1967) y el análisis DAFO (Humphrey, 2005). La Teoría Fundamentada produce explicaciones teóricas de los fenómenos estudiados a través de la inducción, permitiendo así ir de los casos particulares a la generalización (Bunge, 2018). El proceso analítico permite la comparación de datos cuantitativos (frecuencia de las categorías) y cualitativos mediante los distintos tipos de codificación: abierta, axial y selectiva, a partir de los cuales se crean mapas conceptuales con los temas y contenidos principales (Glaser y Strauss, 1967; Guba y Lincoln, 2002). Siguiendo a Strauss y Corbin (1990), esta metodología cumple con los criterios de rigurosidad que exige la investigación científica. Por otro lado, el análisis DAFO (Humphrey, 2005), acrónimo de debilidades, amenazas, fortalezas y oportunidades (Humphrey, 2005), se ha utilizado a posteriori sobre las respuestas obtenidas con el primer tipo de análisis para explorar el equilibrio percibido en la relación de aspectos negativos y positivos, cuyo origen puede ser tanto interno como externo.

\section{Participantes}

Ya que no se cuenta con un censo de la población gitana en las Islas Canarias, se han realizado consultas con informantes claves del Pueblo Gitano como son los pastores evangélicos y los/as responsables del movimiento asociativo, los cuales estimaron que familias con hijos/as con edades comprendidas entre los 6 y los 17 años, puede estar entre unas 180-200 familias. Una vez identificadas, finalmente se pudo contactar con estas, de las cuales aceptaron ser entrevistadas 53 familias de Tenerife, 30 familias de Gran Canaria y 12 familias de Lanzarote, suponiendo un total de 95 familias con hijos e hijas cursando Educación Primaria y Educación Secundaria Obligatoria. La tabla 2 muestra las características sociodemográficas de las familias entrevistadas. 
Tabla 1.

Perfil sociodemográfico de las familias entrevistadas

\begin{tabular}{|c|c|c|c|}
\hline Variable & $\begin{array}{c}\text { Familias de Tenerife } \\
\qquad \begin{array}{c}(n=53) \\
\%\end{array}\end{array}$ & $\begin{array}{c}\text { Familias de Gran } \\
\text { Canaria }(n=30) \\
\%\end{array}$ & $\begin{array}{c}\text { Familias de Lanzarote } \\
\qquad\left(\begin{array}{c}n=12) \\
\%\end{array}\right.\end{array}$ \\
\hline \multicolumn{4}{|l|}{ Género } \\
\hline Mujer & 69,8 & 33,3 & 58,3 \\
\hline Hombre & 30,2 & 66,7 & 41,7 \\
\hline \multicolumn{4}{|l|}{ Edad } \\
\hline 20-29 & 11,3 & 6,7 & 8,3 \\
\hline $30-39$ & 50,9 & 60,0 & 33,3 \\
\hline $40-49$ & 32,1 & 33,3 & 58,3 \\
\hline $50-59$ & 5,7 & 0,0 & 0,0 \\
\hline \multicolumn{4}{|l|}{ Estado civil } \\
\hline Casado/a & 39,2 & 56,7 & 50,0 \\
\hline Pareja de hecho & 45,1 & 33,3 & 33,3 \\
\hline Soltero/a & 11,7 & 0,0 & 8,3 \\
\hline Separado/a & 3,9 & 10,0 & 8,3 \\
\hline Viudo/a & 0,0 & 0,0 & 0,0 \\
\hline \multicolumn{4}{|l|}{ № de hijos/as } \\
\hline 1 & 24,5 & 6,7 & 8,3 \\
\hline 2 & 45,3 & 46,7 & 50,0 \\
\hline 3 & 11,3 & 33,3 & 33,3 \\
\hline 4 & 9,3 & 10,0 & 8,3 \\
\hline 5 & 9,4 & 3,3 & 0,0 \\
\hline \multicolumn{4}{|l|}{ Situación laboral } \\
\hline Desempleado & 68,0 & 53,3 & 16,7 \\
\hline Autónomo & 17,0 & 23,3 & 50,0 \\
\hline Empleado & 7,5 & 13,3 & 33,3 \\
\hline Jubilado/pensionista & 7,5 & 10,0 & 0,0 \\
\hline \multicolumn{4}{|l|}{ Nivel educativo } \\
\hline Sin estudios & 51,0 & 16,7 & 0,0 \\
\hline Primaria & 31,0 & 66,7 & 83,3 \\
\hline ESO & 6,0 & 6,7 & 16,7 \\
\hline FP & 8,0 & 10,0 & 0,0 \\
\hline Bachiller & 4,0 & 0,0 & 0,0 \\
\hline Universitarios & 0,0 & 0,0 & 0,0 \\
\hline
\end{tabular}

Las familias gitanas entrevistadas presentan los siguientes porcentajes de presencia de mujeres, un 69,8\% para la isla de Tenerife y un 58,3\% para Lanzarote, siendo en Gran Canaria mayor la presencia de hombres, un 66,7\%. El rango de edad más frecuente en Tenerife y Gran Canaria es el de 30 a 39 años, contando con más de un 50,0\% en ambos 
casos, mientras que en Lanzarote es el de 40 a 49 años, con un 58,3\%. Es notable que tanto en Gran Canaria como en Lanzarote el estado civil predominante sea el casado/a, superando un 50\%, mientras que en Tenerife es la pareja de hecho con un $45,1 \%$. Las familias entrevistadas suelen ser de dos hijos en todas las islas, superando un 45,0\%, son un 33\% las familias con tres hijos/as en Gran Canaria y Lanzarote, mientras que aquellas con cuatro o cinco hijos/as no superan el 10\%. Llama significativamente la atención que en las islas de Gran Canaria y Tenerife más de un 50\% de las familias estén desempleadas, siendo en Lanzarote un 50\% autónomos y un 33,3\% empleados. Por último, en la isla de Tenerife un 51\% de las personas entrevistadas no han cursado la primaria, mientras que en Gran Canaria un 66,7\% y en Lanzarote un 83,3\% cuentan con estudios primarios. Así mismo, en todas las islas no llega al 5\% las familias que cuentan con formación a nivel de Bachiller y ninguna tiene estudios universitarios.

\section{Instrumentos}

La recogida de información se ha realizado a través de la entrevista semiestructurada, ya que presenta un grado de flexibilidad que puede adaptarse a los entrevistados, con enormes posibilidades para motivar al interlocutor, aclarar términos e identificar ambigüedades (Díaz-Bravo et al., 2013). La primera entrevista semiestructurada cuenta con un guion de preguntas abiertas en la que se profundiza acerca de los deseos, expectativas y celebraciones más importantes de los padres y madres y metas respecto al futuro de sus hijos e hijas. Una vez, procesadas y analizadas las entrevistas, se ha seleccionado solamente a aquellas familias cuyas metas están orientadas al éxito escolar. Para dichas familias ha sido necesario realizar una segunda entrevista semiestructurada con la que a partir de sus respuestas se profundiza en las fortalezas, oportunidades, debilidades y amenazas que perciben. La tabla 2 presenta la estructura básica de ambas entrevistas.

Tabla 2.

Guion de las entrevistas semiestructuradas

\begin{tabular}{|c|c|c|}
\hline & Preguntas & Destinatarios/as \\
\hline \multirow{7}{*}{$\begin{array}{l}1^{\text {a }} \text { Entrevista } \\
\text { semiestructurada }\end{array}$} & $\begin{array}{l}\text { Nos situamos en el futuro de tus hijos/as ¿cómo te gusta- } \\
\text { ría que fuesen sus vidas? }\end{array}$ & 95 familias \\
\hline & $\begin{array}{l}\text { Cuando piensas en tu hijo/a (nombre) concretamente, } \\
\text { ¿cómo crees que será su vida? }\end{array}$ & \\
\hline & ¿Qué lo/a ves haciendo? & \\
\hline & ¿Hablan en su familia acerca del futuro de sus hijos/as? & \\
\hline & ¿A tu familia le preocupa el futuro de tus hijos/as? & \\
\hline & $\begin{array}{l}\text { ¿Crees que a tu comunidad le preocupa los estudios de } \\
\text { tus hijos/as? }\end{array}$ & \\
\hline & $\begin{array}{l}\text { ¿Crees que a la sociedad mayoritaria le preocupa los } \\
\text { estudios de tus hijos/as? }\end{array}$ & \\
\hline
\end{tabular}




\begin{tabular}{llc}
\hline & \multicolumn{1}{c}{ Preguntas } & Destinatarios/as \\
\hline 2. Entrevista se- & ¿Se siente seguro/a de mí mismo/a como padre/madre? & 15 familias \\
miestructurada & ¿Cumple con sus expectativas en lo que se refiere a dar & \\
& apoyo y afecto a sus hijos/as? \\
& ¿Cómo considera que son sus habilidades como padre/ \\
& madre? \\
& ¿Ayuda a sus hijos/as con las tareas de clase? \\
& Cuando tienes un problema con alguno de tus hijos e \\
& hijas, ¿a qué instituciones (colegio, Servicios Sociales, \\
& ONG, asociación de gitanas, etc.) sueles acudir a pedir \\
& ayuda? \\
& ¿Cuáles son los impedimentos del éxito escolar de sus \\
& hijos/as? \\
& ¿Cómo es la convivencia en el centro educativo? \\
& ¿En el centro se respeta a todas las familias y alumno/as, \\
& evitando discriminaciones? \\
& ¿Cómo es la relación con el profesorado? \\
&
\end{tabular}

\section{Procedimiento de obtención de datos}

El estudio se ha llevado a cabo en dos fases. En la primera fase, gracias a la colaboración de los pastores, pastoras y representantes del movimiento asociativo gitano se hizo un listado con las familias que tuviesen hijos e hijas con edades comprendidas entre los 6 a los 17 años. Una vez identificadas, se contactó con los padres y/o madres a quienes se les ofreció la oportunidad de participar en la investigación en la que el tema central sería las familias gitanas de Canarias y el éxito escolar. La primera entrevista semiestructurada se realizó en diversos lugares, domicilios, iglesias y mercadillos. Antes de realizarlas se les informó a los padres y madres que su participación era de carácter voluntario y anónimo, de modo que los datos aportados se utilizarían únicamente para el estudio. Aceptaron firmar el consentimiento informado según el Comité Ético de la Universidad de La Laguna, 53 familias de la isla de Tenerife, 30 familias de Gran Canaria y 12 familias de la isla de Lanzarote.

En la segunda fase, con el primer análisis de los datos obtenidos en la primera fase, se decidió profundizar en aquellas familias cuyos relatos fueran coherentes y orientados al éxito escolar, con la finalidad de visibilizar a las que han dado un salto de calidad en relación con el desarrollo educativo de sus hijos e hijas. Tras informales del motivo de su selección, se volvió a entrevistar a un total de 15 familias: seis familias de Gran Canaria, una de Lanzarote y ocho de Tenerife.

\section{Procedimiento de análisis de datos}

El procesamiento y análisis de los datos se desarrolló en dos fases. Para la primera fase, se transcribieron y ordenaron las entrevistas (primera entrevista semiestructurada); seguidamente el equipo investigador realizó la codificación abierta, la codificación axial y la codificación selectiva, utilizando los resultados para la construcción del 
mapa conceptual que representa la realidad de las familias gitanas de Canarias y su orientación hacia el éxito escolar de sus hijos/as.

Para la segunda fase, se seleccionaron a las familias orientadas al éxito escolar identificadas en la primera fase. Tras el proceso de transcribir, ordenar y codificar las entrevistas de las 15 familias (segunda entrevista semiestructurada), con los resultados de dicha entrevista se ha aplicado el modelo de informe DAFO, lo que ha permitido organizar las respuestas en función de las fortalezas, oportunidades, debilidades y amenazas percibidas. El informe DAFO permite elaborar recomendaciones y tomar decisiones de mejora, ya sea desde las propias familias, como desde los diferentes colectivos, organismos y sociedad en general.

En ambas fases, se han seguido criterios de calidad de la investigación cualitativa (Palacios et al., 2013), como son la revisión de expertos (en la que participaron 5 expertos) y la triangulación interjueces dando un alto grado de acuerdo (87\%). Todo ello se ha realizado mediante el programa ATLAS.ti 7.

\section{Resultados}

\section{Resultados de la primera fase de entrevistas semiestructuradas}

A continuación, se presentan los resultados obtenidos a partir del análisis de las 95 entrevistas semiestructuradas. En primer lugar, se expone el flujograma (Figura 1) que muestra el proceso de selección hasta llegar a las familias que serían nuevamente entrevistadas. De las 95 familias iniciales se excluyeron ocho familias, ya que en sus discursos presentaban ausencia del valor por la educación reglada. También se ha excluido a 46 de ellas, ya que presentan una visión negativa de sus hijos, así mismo se han excluido a 26 familias porque sus discursos presentan incoherencia entre sus deseos, expectativas y celebraciones, siento un total de 15 familias las que muestran mayor coherencia y una orientación hacia el éxito escolar.

En segundo lugar, tras el proceso de codificación abierta, axial y selectiva de los resultados de la primera fase con 95 familias, se ha realizado un mapa conceptual con las distintas orientaciones y posibilidades que presentan las figuras parentales hacia el éxito escolar (Figura $2^{1}$ ). Cabe destacar que 8 figuras parentales están orientadas hacia la tradición cultural con ausencia de valor por la educación reglada, lo que supone una contradicción al éxito escolar de sus hijos/as. Por su parte, 87 figuras parentales están orientadas hacía la preservación de su identidad étnica, el desarrollo personal y socioeconómico, mostrando así la importancia que tiene la formación escolar para la consecución de sus deseos. Ahondando más en el análisis de este grupo, resulta que 44 figuras parentales presentan unas expectativas centradas en el déficit, mientras que 43 de ellas están centradas en las potencialidades, lo que las orienta como candidatas al éxito escolar de sus hijos e hijas. Sin embargo, dando un paso más allá en la contrastación, resulta que 28 figuras parentales del grupo de potencialidades

1 Nota. Las figuras ovaladas incluyen la codificación selectiva y el número de figuras parentales a las que se le aplican, unidos por una línea a las figuras rectangulares que incluyen los códigos abiertos y axiales y el número de las figuras parentales que los presentan. Por su parte, la dirección de las flechas muestra las orientaciones de las figuras parentales hacia el éxito escolar 
presentan contradicciones entre los deseos, las expectativas y las prioridades, es decir, coinciden entre deseos y expectativas, pero carecen de orientaciones que den prioridad a la formación académica. Por tanto, son 15 las figuras parentales que presentan un discurso coherente entre sus deseos, expectativas y prioridades celebraciones más significativas, por lo que se consideran potencialmente orientadas al éxito escolar.

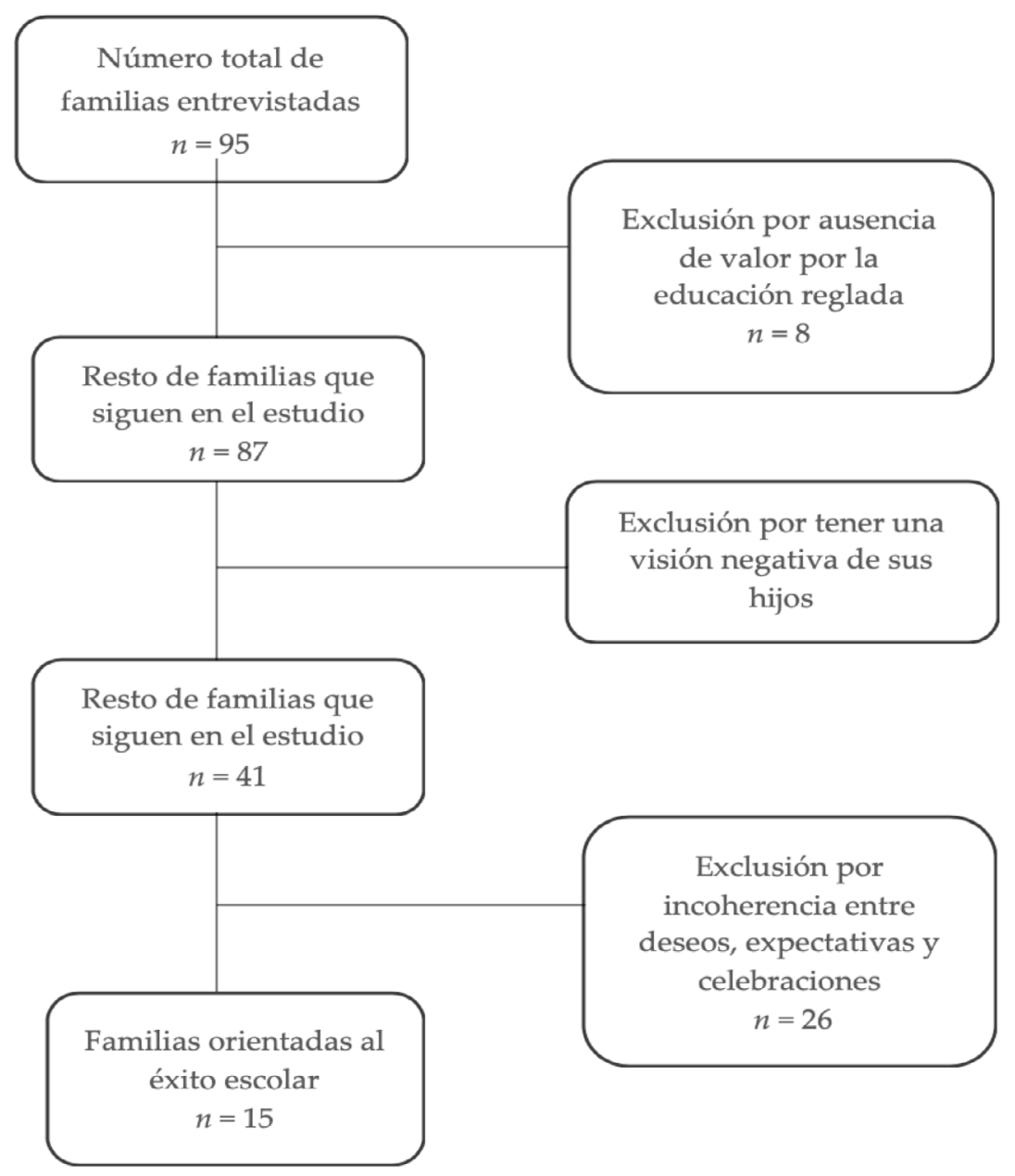

Figura 1. Flujograma de familias orientadas al éxito escolar 


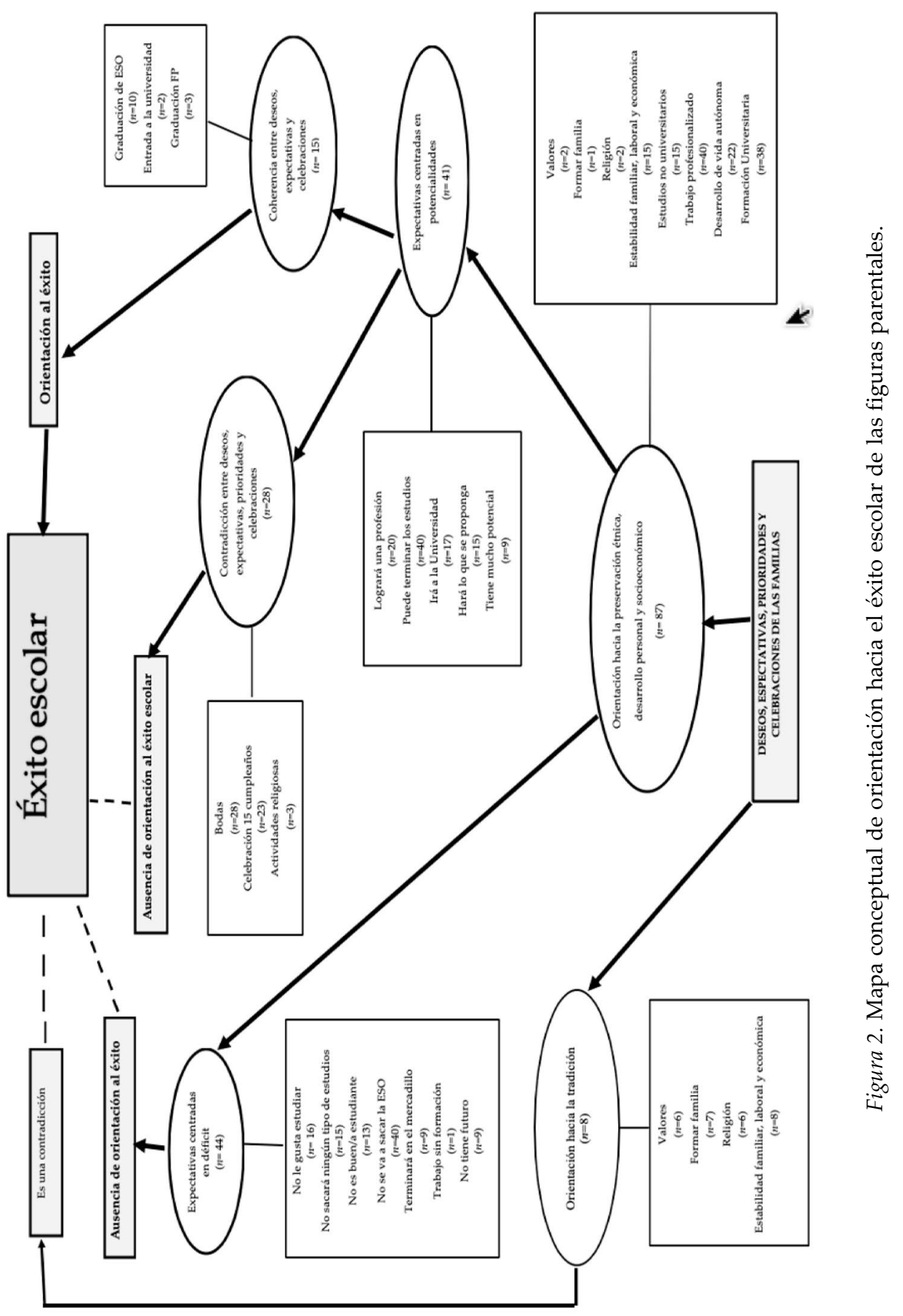




\section{Resultados de la segunda fase de entrevistas semiestructuradas}

En la Tabla 3 se presentan los resultados obtenidos a partir del informe DAFO aplicado a los resultados de la segunda entrevista realizada a las 15 familias seleccionadas de la primera fase.

Tabla 3.

Informe DAFO de las familias orientadas al éxito escolar

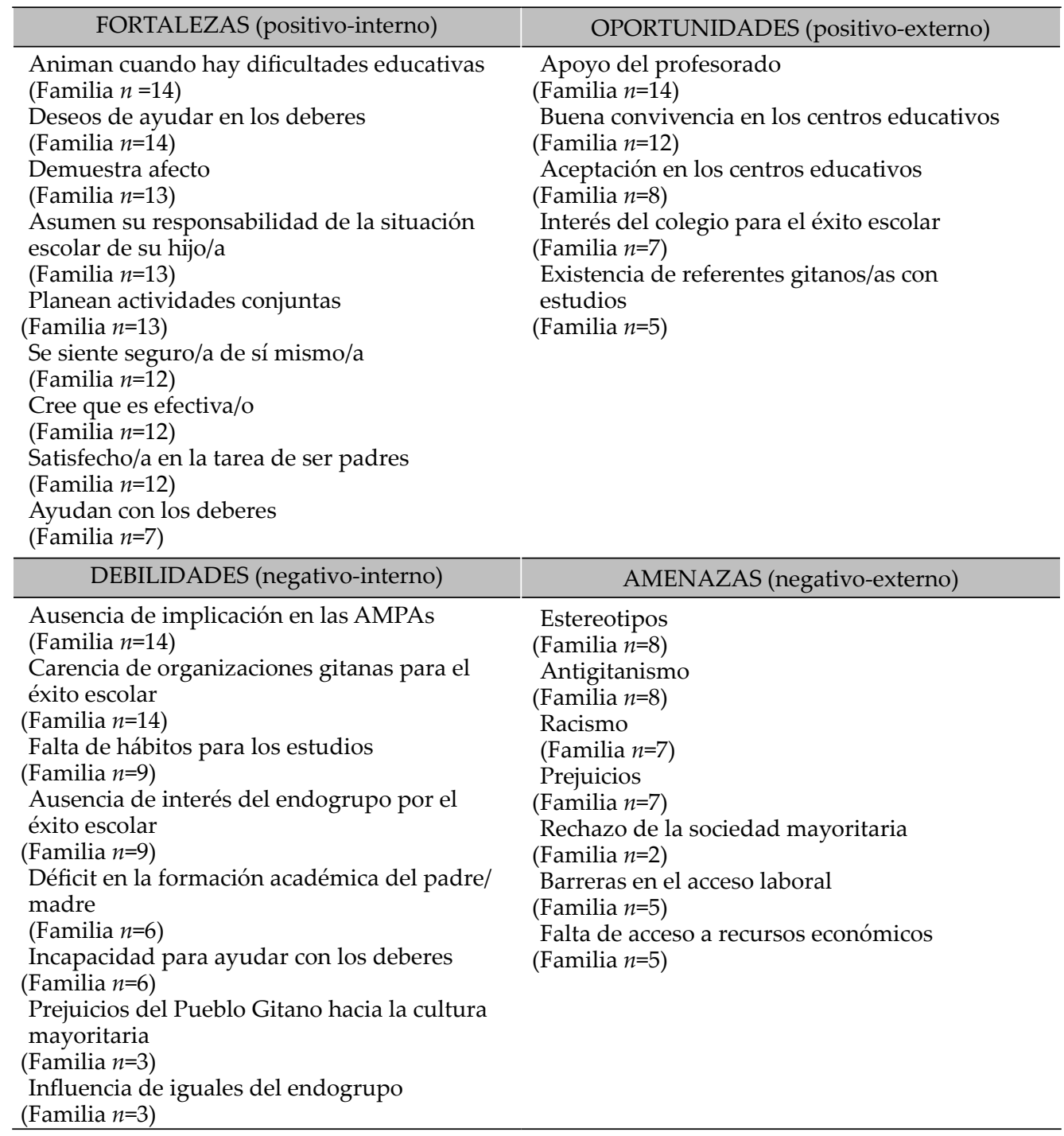

Nota. $n=n$ úmero de familias 
Analizando las respuestas de las figuras parentales a la segunda entrevista según el modelo de informe DAFO se observa que los tipos de fortalezas mencionados (9) apenas son más que los tipos de las debilidades expuestas (8), mientras que los tipos de amenazas (7) son más numerosos que los de las oportunidades (5). Entre el elemento positivo interno, las fortalezas, destaca el deseo de ayudar en la realización de deberes escolares $(n=14)$ y el apoyo emocional que dan a los hijos/as $(n=14)$. Así mismo, las figuras parentales asumen la responsabilidad de la situación de sus hijos/as en los centros educativos $(n=13)$. En lo que se refiere al elemento negativo interno, las debilidades, destaca la ausencia de la implicación en las AMPAs $(n=14)$, así mismo, la carencia de asociaciones gitanas orientadas a favorecer el éxito escolar $(n=14)$. A su vez, la falta de interés del endogrupo también es significativo $(n=9)$, a lo que se suma la falta de hábito personal para los estudios $(n=9)$, lo que demuestra la carencia de dispositivos de apoyo del propio Pueblo Gitano en materia educativa. Por otra parte, hay que destacar que respecto al elemento positivo externo, las oportunidades, casi todas las familias orientadas hacia el éxito escolar cuentan con apoyo del profesorado $(n=14)$, siendo elevado el número de las que tienen buena convivencia en el centro educativo $(n=12)$, elementos claves para el logro del éxito en la formación de sus hijos/ as. Por último, respecto al elemento negativo externo es significativo que el antigitanismo $(n=8)$ junto a los estereotipos $(n=8)$ sean las amenazas más mencionadas que perciben las figuras parentales, a las que se les suma el racismo $(n=7)$ y los prejuicios $(n=7)$. Con menor ocurrencia destacan la falta de acceso a recursos económicos $(n=$ 5) y barreras de acceso al mercado laboral $(n=5)$.

\section{Discusión y conclusiones}

Poniendo en valor la capacidad de resiliencia del Pueblo Gitano, cabe destacar que la familia es su mayor fortaleza siendo el eje principal sobre el que se fundamenta su cultura (Garriga, 2000; Khalfaoui, 2019). Así lo confirman las 95 familias entrevistadas, ya que entre sus expectativas y metas destacan la "formación de familia", la "estabilidad familiar y laboral" y la "preservación de los valores", propios de su cultura. En pleno siglo XXI, los gitanos y gitanas, no solo quieren seguirlo siendo, sino que, además, lo necesitan. Es evidente que las familias gitanas se sitúan entre el arraigo a una cultura milenaria y los desafíos de las sociedades postmodernas. Como define San Román (2005), las familias romaníes viven entre "la necesidad y la agonía de seguir siendo gitano" (p.29) ya que una cierta lectura de sus valores familiares puede también competir con sus necesarios avances y su supervivencia en nuestras sociedades modernas.

El Pueblo Gitano está dando pasos decididos para su inclusión plena, ya que se están dando importantes cambios en la percepción del sistema educativo. El cambio de mentalidad se hace tangible cuando las 87 familias de nuestro estudio desean el éxito escolar, tanto de sus hijos como de sus hijas, ya que se orientan hacía el desarrollo personal y socioeconómico. Sin duda, las familias gitanas de Canarias han superado lo que el modelo ecológico cultural de Ogbu, (1981, citado en Abajo y Carrasco, 2011) llama el "acting white", lo que sería sinónimo de "apayarse". Si bien, hace dos o tres décadas tener un hijo o hija con estudios era visto como una amenaza para la preservación de la identidad gitana, ahora es motivo de orgullo y un prestigio (Abajo 
y Carrasco, 2011). Como evidencian las 87 padres y madres entrevistados, entre sus deseos destacan que sus hijos e hijas realicen al menos "estudios no universitarios", "formación universitaria" y "trabajo profesionalizado"; lo que deviene en la esperanza del logro del "desarrollo de una vida autónoma" que es un gran valor de la cultura gitana. Las familias gitanas son conscientes de que la formación académica no sólo da posibilidades económicas, sino que es un medio de alcanzar la dignidad, autonomía y promoción social (Carmona et al., 2017). El fracaso escolar, no solo supone un hándicap por razones económicas, sino que la falta de formación reduce al mínimo las posibilidades de autonomía, un valor propio de la cultura gitana, dejando a las familias a merced de la asistencia social, lo que supone un atentado contra la autoestima y el orgullo de los matrimonios gitanos, que se han caracterizado por ser capaces de valerse por sí mismos, autónomos y emprendedores (Parra et al., 2017).

Ahora bien, no se debe pasar por alto que 80 figuras parentales de las 95 entrevistadas presenten carencias en la orientación escolar de sus hijos e hijas. De las cuales 46 de ellas tienen expectativas centradas en el déficit, expresando frases como: "a mi hijo no le gusta estudiar", "no se va a sacar la ESO”, "terminará en el mercadillo", etc. Contando a su vez, con 26 padres y madres con discursos contradictorios entre sus expectativas y metas, ya que entre sus celebraciones más importante están "las bodas" "los cumpleaños de quince años" y las "actividades religiosas", con ausencia de todo lo vinculado a lo educativo. Sin embargo, el estudio identifica las características diferenciales de 15 figuras parentales que presentan una coherencia entre expectativas y metas, y cuyo enfoque está centrado en las potencialidades de sus hijos e hijas. Lo que demuestra que es posible mantener una identidad étnica milenaria que cuenta con un enorme capital cultural (Yosso, 2006) centrado en la familia como pilar esencial sobre el que se fundamenta su cultura (Gómez-Alfaro, 2010; Hancock, 2011) y una orientación familiar en la que el desarrollo pleno de las capacidades de la infancia sea prioritario. Como bien indican Abajo y Carrasco (2004), la investigación sociológica, el discurso institucional, y la "tradición escolar" yerran al señalar las divergencias entre la educación escolar y la cultura gitana, lo que no sólo constituye una gravísima imprudencia sino también una total inexactitud desde la evidencia científica. Los resultados del presente estudio se suman a los trabajos realizados por Abajo y Carrasco (2004) y de Cárdenas-Rodríguez et al. (2019), al evidenciar que, tanto en Canarias como en el resto del territorio español, la promoción escolar de la infancia gitana es posible y perfectamente compatible con la preservación y aprovechamiento de su capital cultural; si bien es cierto que no es la regla sino la excepción, el desafió está servido.

A fin de revertir la situación de fracaso escolar de la infancia gitana, las familias deben asumir que la promoción educativa demanda algo más que deseos. Siguiendo a Álvarez et al. (2018), las expectativas parentales son un factor clave para el éxito escolar. De ahí que sea relevante en nuestro estudio que haya 15 familias gitanas con expectativas centradas en potencialidades y priorizando el éxito educativo de sus hijos e hijas. A lo que se le suma, según los resultados del informe DAFO, que estas familias realizan atribuciones internas al percibir que sus fortalezas están basadas en las propias capacidades parentales, tales como la capacidad de planificar actividades, la demostración de afecto y la satisfacción en la tarea de ser padre y madre. Han experimentado una buena relación con la escuela y se interesan y apoyan en las tareas escolares. Con esta 
caracterización el estudio no pretende presentar a "la familia modelo", sino más bien, visibilizar familias referentes, las cuales ponen en acción una serie de principios que hacen posible el éxito escolar en la infancia gitana. Saben que no es suficiente escolarizar y aspirar a su graduación sin proporcionarles el apoyo familiar necesario. Los niños y niñas necesitan que se les propongan metas altas pero alcanzables, desafíos que les permitan descubrir sus capacidades, la libertad de tomar sus propias decisiones sin temor a equivocarse, así como sentirse valorados independientemente de los resultados que puedan obtener (Baños-Gil, 2010). Por lo que es imprescindible que los padres y madres de etnia gitana, al igual que todos los que pretendan apoyar el logro educativo de sus hijos e hijas, se esfuercen en creer, estimular y centrarse en sus potencialidades.

Es significativo que las 15 figuras parentales realicen atribuciones internas al percibir, entre sus debilidades, la ausencia de interés del propio endogrupo por el éxito escolar de la infancia gitana. Como evidencian las investigaciones de Saavedra y Villalta (2008, citado en Torres y Ruiz, 2012) y Villalobos Cano (2009, citado en Torres y Ruiz, 2012), las figuras parentales que logran el éxito escolar de sus hijos e hijas en nuestro estudio, incluso en contextos adversos de amenazas y falta de oportunidades, presentan atribuciones internas, ya que consideran que el éxito de dichos logros se debe a las acciones que ellos realizan. Por consiguiente, el Pueblo Gitano, en lo que a la educación reglada se refiere, necesita de tales atribuciones internas tanto para valorar sus fortalezas como para superar las debilidades. Este es el modo de salir de la pasividad propia de las atribuciones a factores externos macro-estructurales, que deviene en una actitud de indefensión aprendida (Seligman y Maier, 1985), dejando su futuro en manos del asistencialismo y la caridad.

Si bien es cierto que el Pueblo Gitano está cambiando su visión sobre la educación reglada, no es suficiente que esté bien visto poseer un título académico, sino que, además, se debe asumir el compromiso de la propia comunidad para que cada niño y niña gitana lo logre. Cobra vital importancia que en la dimensión externa de amenazas del informe DAFO, sean precisamente las familias que están orientadas hacia el éxito escolar de sus hijos las que aun perciban antigitanismo, prejuicios, estereotipos, rechazo de la sociedad mayoritaria y racismo. En el imaginario popular aún pervive la imagen estereotipada del gitano, fruto de seis siglos marcados por la represión, el exterminio y la exclusión (Gómez-Alfaro, 2010; Jiménez-González y Laparra, 2016). Por lo que la sociedad española necesita ahondar en las reformas que provoquen un cambio en la percepción de la gitanidad que ofrezca oportunidades para que las familias gitanas que inician su recorrido encuentren un entorno favorable. Siguiendo a Velasco (2007), la discriminación étnico-racial debe dejar de ser tratada como un racismo de importancia secundaria, enfrentándose con un marco legal que sancione tanto social como políticamente aquellos comportamientos que lo manifiesten. Asimismo, los centros educativos deben impulsar los procesos necesarios para garantizar la inclusión y participación del alumnado gitano en la vida del centro, de ahí que sea clave la promoción de la cultura gitana, para que se conozca la influencia del Pueblo Gitano tanto en la historia de España, en el idioma español, así como en el arte desarrollado en la Península Ibérica (Fernández et al., 2015).

En conclusión, desde una perspectiva ecosistémica es indudable que la plena inclusión educativa de los niños y niñas gitanos depende de múltiples factores sociohistóricos, 
económicos y culturales que interactúan y se retroalimentan dando lugar a una realidad compleja que, a través del sistema familiar y escolar en mutua relación, moldean la vida del individuo (Carmona et al., 2019). Sin embargo, esa misma perspectiva enfatiza que los factores proximales (i.e., familia y escuela), cercanos al individuo, tienen un mayor peso que los factores distales cuando se trata de moldear sus procesos de desarrollo. Por tanto, es importante incidir en propiciar estos procesos de cambio en el seno de las familias y las escuelas. Los resultados del presente estudio evidencian que 15 familias gitanas de Canarias, libres de un escenario de marginalidad, han dado un paso hacia delante en la valoración y promoción de la formación académica de sus hijos e hijas. Son familias con figuras parentales referentes, capaces de tener coherencia entre sus expectativas y metas, cuyos deseos, celebraciones y prioridades son congruentes con dichas metas; a lo que se le suma la destreza para hacer frente a los desafíos propios de cada día, la capacidad de creer que un futuro mejor es posible para sus hijos e hijas y su lucha constante para conseguirlo. Estas familias nos enseñan una gran lección: la España cohesionada e inclusiva seguirá siendo una utopía mientras siga pendiente la normalización del éxito escolar de la infancia gitana.

\section{Referencias}

Abajo, J. E. \& Carrasco, S. (Eds.) (2004). Experiencias y trayectorias de éxito escolar de gitanas y gitanos en España. Ministerio de Igualdad y Ministerio de Educación Política Social y Deporte.

Abajo, J. E. \& Carrasco, S. (2011). La situación escolar del alumnado de minorías étnicas: el modelo explicativo ecológico-cultural de John Ogbu. Revista de Pensament $i$ Anàlisi, 11, 71-92.

Álvarez, E., Zaragoza, M., Blanch, T., Mayayo, E., \& Romaní, J. (2018). Condicionantes del éxito y fracaso escolar en contextos de bajo nivel socioeconómico. REXE. Revista de Estudios y Experiencias en Educación, 2(1), 75-94.

Baños-Gil, I. (2010). El efecto Pigmalión en el aula. Revista Digital Innovación y Experiencias Educativas, 28, 1-9.

Bornstein, M. H. (2002). Handbook of parenting: Practical issues in parenting. Erlbaum.

Bunge, M. (2018). La ciencia: su método y su filosofía. Laetoli.

Bronfenbrenner, U. (1987). La ecología del desarrollo humano. Paidós.

Cárdenas-Rodríguez, R., Terrón-Caro, T., \& Gimeno, M. (2019). Educación Primaria y alumnas gitanas. Análisis de las barreras sociales en contextos de exclusión. Revista de Investigación Educativa, 37(1), 75-91. https://doi.org/10.6018/rie.37.1.326221

Carmona, M., González, J., \& Soria, A. (2017). Gitanos en la Universidad: Un estudio de caso de trayectorias de éxito en la Universidad de Sevilla. Revista de Educación, $377,187-211$.

Carmona-Santiago, J., García, M., Máiquez, M. L., \& Rodrigo, M. J. (2019). El impacto de las relaciones entre la familia y la escuela en la inclusión educativa de alumnos de etnia gitana. Una revisión sistemática. Multidisciplinary Journal of Educational Research, 9(3), 319-348. http://dx.doi.org/10.17583/remie.2019.4666

Collet, J., \& Tort, A. (2011). Famílies, escola i èxit. Millorar Els Vincles per Millorar Els Resultats. Fundació Jaume Bofill. 
Consejo de Europa (2006). Recomendación (2006) 19 del Comité de Ministros a los Estados Miembros sobre Políticas de Apoyo a la Parentalidad Positiva. Consejo de Europa.

Díaz-Bravo, L., Torruco-García, U., Martínez-Hernández, M., \& Varela-Ruiz, M. (2013). La entrevista, recurso flexible y dinámico. Investigación en Educación Médica, 2(7), 162-167.

Enseñanza CCOO (2019). Abandono temprano de la educación y la formación y fracaso escolar: diagnostico y propuestas. Federación de Enseñanza CCOO.

Espinal, I., Gimeno, A., \& González, F. (2006). El enfoque sistémico en los estudios sobre la familia. Revista internacional de sistemas, 14(4), 21-34.

Fernández-Jiménez, D. (2018). Una respuesta a la cuestión gitana. Reflexiones jurídicoconstitucionales sobre una minoría cultural española. Universidad de Córdoba, UCOPress.

Fernández, H., Jiménez, N., \& Motos, I. (2015). Guía de recursos contra el antigitanismo. Federación Autonómica de Asociaciones Gitanas de la Comunidad Valenciana (FAGA).

Flecha, R., \& Soler, M. (2013). Turning difficulties into possibilities: Engaging Roma families and students in school through dialogic learning. Cambridge Journal of Education, 43(4), 451-465. https://doi.org/10.1080/0305764X.2013.819068

Fundación Secretariado Gitano (2000). La educación del niño gitano en Europa. Revista Bimestral de La Asociación Secretariado General Gitano, 8-10. https://bit.ly/2Zwy9NT

Fundación Secretariado Gitano. (2019). Estudio comparado sobre la situación de la población gitana en España en relación al empleo y la pobreza 2018. Fundación Secretariado Gitano.

Garriga, C. (2000). Els gitanos de Barcelona. Àrea de Serveis Socials.

Garriga, C., \& Carrasco-Calvo, S. (2011). Tradición y cambio en la vida de los gitanos. O Tchatchipen, 97, 30-36.

Geddes, H. (2010). El apego en el aula: relación entre las primeras experiencias infantiles, el bienestar emocional y el rendimiento escolar (Vol. 269). Graó.

Giménez-Adelantado, A. (2003). La escolarización de la infancia gitana en 167 poblaciones de Andalucía (Vol. 8). Publicacions de la Universitat Jaume I.

Giménez-Cortés, A., Comas, D., \& Carballo, A. (2019). Identidad y origen del pueblo gitano. International Journal of Roma Studies, 1(2), 159-184.

Glaser, B. \& Strauss, A. (1967). The discovery of Grounded Theory: Strategies for qualitative research. Aldine Publishing Company.

Gómez-Alfaro, A. (2010). Escritos sobre gitanos. Asociación de Enseñantes con Gitanos.

Guba, E. \& Lincoln, Y. (2002). Paradigmas en competencia en la investigación cualitativa. En C. Denman y J. Haro (Comps.), Por los rincones: antología de métodos cualitativos en la investigación social (113 - 146). El colegio de la sonora.

Hancock, I. (2011). Romani origins and romani identity: A Reassessment of the arguments. Cambridge Scholars' Press.

Humphrey, A. S. (2005). SWOT analysis. Long Range Planning, 30, 46-52.

Jiménez-González, N. (2018). La historia del pueblo gitano: memoria e inclusión en el curriculum educativo. Drets. Revista Valenciana de Reformes Democràtiques, (2), 113-130.

Jiménez-González, N. \& Laparra, D. (2016). Santipen aj Rroma: Desigualdad en Salud y comunidad gitana. Análisis y propuestas desde el ámbito sociosanitario. Publicacions de la Universitat d'Alacant. 
Khalfaoui, A. (2019). Continuidades y cambios en la identidad de la mujer gitana. Un estudio de caso. International Journal of Roma Studies, 1(2), 185-203.

Kiernan, K. E., \& Mensah, F. K. (2011). Poverty, family resources and children's early educational attainment: the mediating role of parenting. British Educational Research Journal, 37(2), 317-336.

Kottak, P. (2011). Antropología cultural. McGraw-Hill.

Laparra, M. (2011). Diagnóstico social de la comunidad gitana en España. Un análisis contrastado de la Encuesta del CIS a Hogares de Población Gitana 2007. Ministerio de Sanidad, Política Social e Igualdad.

Labin, A., Brenlla, M., \& Taborda, A. (2019). Estudio preliminar sobre la relación entre el nivel educativo de la madre y los índices comprensión verbal y velocidad de procesamiento del WISC-IV. Revista de Psicología, 11(21), 35-45. https://bit.ly/3jbXILR

López, F. (2008). Necesidades en la infancia y en la adolescencia. Respuesta familiar, escolar y social. Pirámide.

López-Ros, S. (2011). Gitanidad: otra manera de ver el mundo. Editorial Kairós.

Macías, F. (2017). Contribución del Pueblo Gitano para luchar contra la Pobreza y el Antigitanismo a través de su participación en Actuaciones Educativas de Éxito. [Tesis doctoral no publicada]. Universidad de Barcelona.

Marcos, M., Prada, A., Brotons, M. \& Sabbatella, P. (2017). Análisis de contenido y análisis DAFO en una investigación cualitativa sobre la Musicoterapia en España. Investigación Cualitativa en Ciencias Sociales, 3, 403-412. https://bit.ly/2WpJXzD

Marchesi, A. \& Martin, E. (2003). Evaluación de la educación secundaria. Fotografía de una etapa polémica. Instituto IDEA, SM.

Martínez, C., Redondo, R., Fabra, M., Núñez, A. \& Martín, M. (2010). Influencia del Nivel Educativo de los Padres en el Rendimiento Académico de los Estudiantes de ADE. Un enfoque de Género. AEDE, 5(1), 1273-1294. https://bit.ly/399o50v

Matamoros, J., Archila, L. \& Güichá-Duitama, Á. (2017). Relación entre sintomatología depresiva y cohesión familiar en adolescentes de una institución educativa de Boyacá. Psicogente, 20(38), 296-307. https://doi.org/10.17081/psico.20.38.2550

Ministerio de Sanidad, Servicios Sociales e Igualdad (2012). Estrategia Nacional para la Inclusión Social de la Población Gitana en España 2012-2020. Ministerio de Sanidad, Servicios Sociales e Igualdad.

Ministerio de Sanidad Servicios Sociales e Igualdad (2016). Segunda Encuesta Nacional de Salud a Población Gitana, 2014. Ministerio de Sanidad, Servicios Sociales e Igualdad.

Palacios, B., Sanchez, M.C., \& Gutiérrez, A. (2013). Evaluar la calidad en la investigación cualitativa. Guías o checklists. En M. Pacheco, M. V. Mariño y T. González (coords.). $2^{\circ}$ Congreso Nacional sobre Metodología de la Investigación en Comunicación, pp. 581-596. Valladolid: Facultad de Ciencias Sociales, Jurídicas y de la Comunicación. https:// bit.ly/2ChAGTn

Parra, I., Álvarez-Roldan, A., \& Gamella, J. F. (2017). Un conflicto silenciado: Procesos de segregación, retraso curricular y abandono escolar de los adolescentes gitanos. Revista de Paz y Conflictos, 10(1), 35-60.

Quecedo, R., \& Castaño, C. (2002). Introducción a la metodología de investigación cualitativa. Revista de Psicodidáctica, 14, 5-39. 
Rodrigo, M. J., Máiquez, M. L., \& Martín, J. C. (2010). Parentalidad positiva y políticas locales de apoyo a las familias. Orientaciones para favorecer el ejercicio de las responsabilidades parentales desde las corporaciones locales. Ministerio de Sanidad y Política Social.

Rodrigo, M. J., Máiquez, M. L., Martín, J. C., Byrne, S., \& Ruiz, B. (2015). Manual práctico de parentalidad positiva. Síntesis.

Salinas, J. (2009). Un viaje a través de la historia de la escolarización de las gitanas y gitanos españoles. Anales de historia contemporánea, 25, 167-188. https://bit.ly/2ZwZVKi

Salinas, J. (2015). Una aproximación a la historia de la escolarización de las gitanas y gitanos españoles (2a Parte: Siglos XX y XXI). Cabás, 13, 92-112. https://bit.ly/2CG78P4

San Román, T. (2005). La necesidad y la agonía de seguir siendo gitanos. En A. González y J. Molina (Coords.) Memorias de papel de la Asociación de Enseñantes con Gitanos (pp.7-18). Asociación de Enseñantes con Gitanos.

Seligman, M. \& Maier, S. (1985). Indefensión aprendida. Madrid: Debate.

Sime, D., Fassetta, G., McClung, M., \& Council, G. C. (2014). Roma families' engagement with education and other services in Glasgow. University of Strathclyde.

Strauss, A. \& Corbin, J. (1990). Basics of Qualilative Research: Grounded Theory, procedures and techniques. Sage Publications.

Torres, M. de L., \& Ruiz, A. (2012). Motivación al logro y el locus de control en estudiantes resilientes de bachillerato del Estado de México. Psicología Iberoamericana, 20(2), 49-57.

Velasco, S. (2007). El racismo y las tres formas básicas de combatirlo. Cultura y Representaciones Sociales, 2(3), 131-150. https://bit.ly/301rB3O

Yosso, T.J. (2006). Whose culture has capital? A critical race theory discussion of community cultural wealth. Race, Ethinicity and Education, 8(1), 69-91. https://doi. org/10.1080/1361332052000341006

Fecha de recepción: 27 de octubre de 2019.

Fecha de revisión: 29 de noviembre de 2019.

Fecha de aceptación: 24 de agosto de 2020. 